\title{
On the Weak Grothendieck Group of a Morphic Ring and its Representations
}

\author{
Sorokin O.S. \\ Department of Algebra and Logic, Faculty of Mechanics and Mathematics, Ivan Franko National University of Lviv, 79059, Lviv, Ukraine
}

Copyright (C)2015 Horizon Research Publishing All rights reserved.

\begin{abstract}
The K-theoretical aspect of the commutative mophic rings is established using the arithmetical properties of the morphic rings in order to obtain a ring of all Smith normal forms of matrices over the morphic ring. The internal structure and basic properties of such rings are discussed as well as their presentations by the Witt vectors. In a case of a commutative von Neumann regular rings the famous Grothendieck group $K_{0}(R)$ obtains the alternative description.
\end{abstract}

Keywords Grothendieck Group, Morphic Ring, K-theory, Witt Ring, von Neumann Regular Ring, Smith Normal Form, Bezout Ring, Principal Ideals

\section{Mathematics Subject Classification (2010) 19A49, 13D15, 13F35}

\section{Introduction}

In [10] it is proved that for any element $a$ of a commutative morphic ring $R$ there is an element $b \in R$ such that the ideals $a R, b R$ coincides with the annihilators $\operatorname{Ann}(b), \operatorname{Ann}(a)$. Therefore, one can make a partition on the pairs of the set of all principal ideals such that any element of such of this partition is uniquely determined by some pair of principal ideals. So, the structure of principal ideals determines all the properties of the morphic rings.

We will construct an analogue of the Grothendieck group $K_{0}(R)$ of a ring $R$ using the principal ideals instead of the finitely generated projective $R$-modules. Such abelian group, that is denoted as $K_{0}^{\prime}(R)$ and is called a weak Grothendieck group of a ring $R$, becomes a ring if we define a product of two elements of this group using the tensor product of principal ideals. Moreover, the elements of such ring can be interpreted as classes of equivalence of the Smith normal forms of the matrices over a ring $R$.

In order to obtain a convenient way for the multiplication and the addition for the elements of $K_{0}^{\prime}(R)$ we will establish the connection with the subring $W^{\prime}(G(R))$ of the ring of Witt vectors over some ring. As a direct consequence it will be proved that the functors $K_{0}^{\prime}$ and $W^{\prime} G$ are naturally equivalent.
In a case of a commutative von Neumann regular ring the structure of $K_{0}^{\prime}(R)$ becomes simpler and, as a consequence, $K_{0}^{\prime}(R)$ and the usual Grothendieck group $K_{0}(R)$ coincide.

The main motivation of these investigations is that in [11]it is proved that a commutative Bezout domain is an elementary divisor ring if and only if any quotient ring $R / a R$ is so, where $a$ is an arbitrary nonzero element of $R$. Since any finite homomorphic image of a commutative Bezout domain $R$ is a morphic ring [14] then the studies of the ring $K_{0}^{\prime}(R / a R)$ become related to the famous elementary divisor ring problem [8].

\section{Preliminaries}

All the rings considered in the article are supposed to be commutative with the nonzero identity element. By the semiring $R$ we mean a set that satisfies all axioms of a ring except the obligatory existence of additive inverses. The subsemiring $S$ of a semiring $R$ is defined as a subset that is a semiring itself with respect to the addition and multiplication induced from $R$. The simplest examples of semirings are nonnegative integers, set of all subsets of some finite set with respect to the union and intersection operations. For more information we refer to [3]. Let $U(R)$ be a set of all invertible elements of a ring $R$. By the Jacobson radical $J(R)$ of a ring $R$ we mean the set $J(R)=\{x \in R \mid \forall a \in R: 1-a x \in U(R)\}$, and the nilradical $N i l(R)$ is defined as an ideal of all nilpotent elements of a ring $R$. A ring $R$ is called a reduced ring if $\operatorname{Nil}(R)=\{0\}$.

Suppose that $A$ is a subset in a ring $R$. A set $\operatorname{Ann}(A)=$ $\{x \mid A x=0\}$ is called an annihilator of a set $A$. If an element $a \in R$ is a divisor of an element $b \in R$ then we will write $a \mid b$.

We start with recalling of some definitions and facts that we will need below in our proofs.

\section{Definition 1.}

1) If any finitely generated ideal of a ring $R$ is principal then a ring $R$ is said to be a Bezout ring.

2) We say that a rectangular matrix $A$ over a ring $R$ admits canonical diagonal reduction if there are two invertible matrices $P, Q$ of the appropriate sizes such that the matrix

$$
P A Q=D=\left(d_{i}\right)
$$

is a diagonal matrix with an additional condition: for all indices we have the inclusion of the ideals $d_{i+1} R \subseteq d_{i} R$. 
3) If every row matrix $(a, b)$ (column matrix $\left.(a, b)^{T}\right)$ admits canonical diagonal reduction then we say that $R$ is a right (left) Hermite ring.

4) If every matrix over a ring $R$ admits canonical diagonal reduction then $R$ is said to be an elementary divisor ring.

It is not odd to say that any elementary divisor ring is an Hermite one, and the Hermite rings are Bezout rings. An examples of Bezout rings are all principal ideal rings, the rings of real-valued functions over the topological F-space, all polynomial rings over von Neumann regular rings. As more narrow class elementary divisor rings include the ring of entire functions, the ring of algebraic integers, all adequate rings, the Bezout rings of stable range 1. For more information see [13].

Definition 2. We say that a ring $R$ has the stable range 1 if for any elements $a, b \in R$ the equality $a R+b R=R$ implies that there is some $x \in R$ such that $(a+b x) R=R$ [1].

Remark that the last definition is left-right symmetric due to [12].

\section{Definition 3}

A ring $R$ is called a morphic ring if for any $a \in R$ there is an $R$-module isomorphism $R / a R \cong \operatorname{Ann}(a)$ [10].

Here is a Nicholson's criterion for a morphic ring.

Theorem [10].

The following statements are equivalent for a ring $R$ :

a) $R$ is a morphic ring;

b) For any $a \in R$ one can find $b \in R$ such that $\operatorname{Ann}(a)=$ $b R, \operatorname{Ann}(b)=a R$;

c) For any $a \in R$ one can find $b \in R$ such that $\operatorname{Ann}(a)=$ $b R, \operatorname{Ann}(b) \cong a R$.

Also in [10] it is proved every the commutative von Neumann regular ring is a morphic one. In addition it is useful to mention that a pair $(a, b)$ of elements of a ring $R$ in the previous theorem is called a morphic pair and this fact will be denoted as $a R \sim b R$, since any morphic pair is determined by the pair of some principal ideals, but not elements.

Definition 4. An element $a$ of a ring $R$ is said to be a von Neumann regular element if there is some $b \in R$ such that $a b a=a$. If all elements of a ring $R$ are von Neumann regular then $R$ is called a von Neumann regular ring.

The finite direct sums of fields, boolean rings, and rank rings are examples of von Neumann regular rings. For more details see [4].

\section{Weak Grothendieck group}

Let $R$ be a commutative morphic ring. We will try to construct an analogue $K_{0}^{\prime}(R)$ of the Grothendieck group $K_{0}(R)$ considering the isomorphism classes of the finite direct sums of the principal ideals of $R$ as the basic objects and using some ideas from [9].

Let $\Delta(R)=\left\{a_{1} R \oplus \ldots \oplus a_{n} R \mid a_{1}, \ldots, a_{n} \in R\right\}$ be a set of all finite direct sums of the principal ideals of $R$. Then we consider a relation " $\sim$ " on the set $\Delta(R)$ defined as

$$
g_{1} \sim g_{2} \Leftrightarrow g_{1} \cong g_{2}
$$

for $g_{1}, g_{2} \in \Delta(R)$. Then let $F(R)$ be a free abelian group generated by the set $\Delta(R) / \sim$. Since every element of $\Delta(R) / \sim$ is in the one-to-one correspondence with the set of all finite diagonal matrices of $R$ and by [8] every diagonal matrix $D$ is equivalent to its Smith normal form (shortly SNF), so in any class of the equivalent elements in $\Delta(R)$ we can choose some SNF that represents this class in $\Delta(R) / \sim$. In fact, one can consider $F(R)$ as a free abelian group generated by the classes of equivalence of all SNF of the matrices over $R$. The elements of the set $\Delta(R) / \sim$ we will denote as $\operatorname{SNF}(g)$, where $g \in \Delta(R)$.

Definition 5. The quotient group $K_{0}^{\prime}(R)$ of a free abelian group $F(R)$ by the subgroup generated by all expressions of the form $\operatorname{SNF}(g)+\operatorname{SNF}\left(g^{\prime}\right)-\operatorname{SNF}\left(g \oplus g^{\prime}\right)$ we will call a weak Grothendieck group of a morphic ring $R$. The elements of $K_{0}^{\prime}(R)$ will be denoted as $[g]$.

In other words, $K_{0}^{\prime}(R)$ is an abelian group of all classes of isomorphic finite direct sums of principal ideals of a morphic ring $R$ with the following property:

$$
[g]+\left[g^{\prime}\right]=\left[g \oplus g^{\prime}\right]
$$

for any $[g],\left[g^{\prime}\right] \in K_{0}^{\prime}(R)$.

Remark, that $\operatorname{SNF}\left(a_{1} R \oplus \ldots \oplus a_{n} R\right)=\operatorname{SNF}\left(b_{1} R \oplus \ldots \oplus b_{m} R\right)$ in $\Delta(R) / \sim$ if and only if

$$
\bigoplus_{i=1}^{n} a_{i} R \cong \bigoplus_{j=1}^{m} b_{j} R
$$

and

$$
\sum_{j=1}^{m} \operatorname{SNF}\left(g_{j}\right)=\sum_{k=1}^{l} \operatorname{SNF}\left(g_{k}^{\prime}\right)
$$

in $F(R)$ if and only if $m=l$ and there is a permutation $\pi \in$ $S_{m}$ such that $\forall j: g_{j} \cong g_{\pi(j)}^{\prime}$.

Lemma 1. Two elements $[g],\left[g^{\prime}\right] \in K_{0}^{\prime}(R)$ are equal if and only if

$$
g \oplus X \cong g^{\prime} \oplus X,
$$

for some $X=a_{1} R \oplus \ldots \oplus a_{n} R$, where $a_{1}, \ldots, a_{n} \in R$.

Proof. Suppose that $[g]=\left[g^{\prime}\right]$. Then

$$
\begin{aligned}
\operatorname{SNF}(g) & -\operatorname{SNF}\left(g^{\prime}\right)=\sum_{i}\left(\operatorname{SNF}\left(x_{i}\right)+\operatorname{SNF}\left(y_{i}\right)-\operatorname{SNF}\left(x_{i} \oplus y_{i}\right)\right)- \\
& -\sum_{j}\left(\operatorname{SNF}\left(x_{j}^{\prime}\right)+\operatorname{SNF}\left(y_{j}^{\prime}\right)-\operatorname{SNF}\left(x_{j}^{\prime} \oplus y_{j}^{\prime}\right)\right),
\end{aligned}
$$

for some $\operatorname{SNF}\left(x_{i}\right), \operatorname{SNF}\left(y_{i}\right), \operatorname{SNF}\left(x_{j}^{\prime}\right), \operatorname{SNF}\left(y_{j}^{\prime}\right) \in F(R)$.

After placing the summands with negative signs to the another part of the equality and using the previous remark we obtain that

$$
g \oplus X \cong g^{\prime} \oplus X,
$$

where $X=\left(\bigoplus_{i}\left(x_{i} \oplus y_{i}\right)\right) \oplus\left(\bigoplus_{j}\left(x_{j}^{\prime} \oplus y_{j}^{\prime}\right)\right) \in \Delta(R)$.

Conversely, if $g \oplus X \cong g^{\prime} \oplus X$ then $\operatorname{SNF}(g \oplus X)=\operatorname{SNF}\left(g^{\prime} \oplus\right.$ $X)$ in $\Delta(R) / \sim$. Hence $[g \oplus X]=\left[g^{\prime} \oplus X\right]$ in $K_{0}^{\prime}(R)$ implies that $[g]+[X]=\left[g^{\prime}\right]+[X]$ and $[g]=\left[g^{\prime}\right]$. The lemma is proved.

In any case the computations that involves the elements of the group $K_{0}^{\prime}(R)$ rise to the expressions with the principal ideals. So the first thing we need to know is: how the preimages of the equal elements of $K_{0}^{\prime}(R)$ can be described in the terms of $R$ ?

Lemma 2. Let $R$ be a morphic ring and $A, B, x R \in \Delta(R)$ and $A, B$ are reduced to the SNF. Then

$$
A \oplus x R \cong B \oplus x R \Leftrightarrow A=B .
$$


Proof. Suppose that $A=a_{1} R \oplus \ldots \oplus a_{n} R, B=b_{1} R \oplus \ldots \oplus$ $b_{n+m} R, a_{1} R \supseteq \ldots \supseteq a_{n} R, b_{1} R \supseteq \ldots \supseteq b_{n+m} R$. By [6] if $A_{1}$ and $B_{1}$ are the SNF of $A \oplus x R$ and $B \oplus x R$ then $A_{1}=B_{1}$. So we are going to compute explicitly SNF $A_{1}$ and $B_{1}$ using the Fitting invariants.

For the simplification of the notations in the proof below we will write $a+b$ and $a b$ for $a R+b R$ and $a R \cap b R$ respectively. The ordering " $\leq$ " corresponds to the natural inclusion of the sets.

After computing the Fitting invariants the normal forms $A_{1}$ and $B_{1}$ are

$$
\begin{gathered}
A_{1}=\left(a_{1}+x\right) \oplus\left(a_{2}+a_{1} x\right) \oplus \ldots \oplus\left(a_{n}+a_{n-1} x\right) \oplus\left(a_{n} x\right) \\
B_{1}=\left(b_{1}+x\right) \oplus\left(b_{2}+b_{1} x\right) \oplus \ldots \oplus\left(b_{n}+b_{n-1} x\right) \oplus \\
\oplus\left(b_{n+1}+b_{n} x\right) \oplus \ldots \oplus\left(b_{n+m-1}+b_{n+m} x\right) \oplus\left(b_{n+m} x\right)
\end{gathered}
$$

From the equality $A_{1}=B_{1}$ we obtain the system of the principal ideal equations:

$$
\left\{\begin{array}{l}
b_{1}+x=a_{1}+x \\
b_{2}+b_{1} x=a_{2}+a_{1} x \\
\cdots \\
b_{n}+b_{n-1} x=a_{n}+a_{n-1} x \\
b_{n+1}+b_{n} x=a_{n} x
\end{array} \quad,\left\{\begin{array}{l}
b_{n+2}+b_{n+1} x=0 \\
\ldots \\
b_{n+m-1}+b_{n+m} x=0 \\
b_{n+m} x=0
\end{array}\right.\right.
$$

From the equation $b_{n+2}+b_{n+1} x=0$ we obtain that $b_{n+2}=0$ and so $b_{n+2}=\ldots=b_{n+m}=0$. As $b_{n+1}+b_{n} x=a_{n} x$ we conclude that $b_{n+1} \leq x$ and $b_{n} x=a_{n} x$.

Multiplying (in fact intersecting!) by $a_{n}$ the equation $b_{n}+$ $b_{n-1} x=a_{n}+a_{n-1} x$ we obtain

$$
\begin{gathered}
a_{n}+a_{n} x=a_{n} b_{n}+a_{n} b_{n-1} x= \\
=a_{n} b_{n}+b_{n} b_{n-1} x=a_{n} b_{n}+b_{n} x \leq b_{n} .
\end{gathered}
$$

But $a_{n}=a_{n}+a_{n} x$ and so $a_{n} \leq b_{n}$. Analogously, multiplying the same equation by $b_{n}$ we will have that $b_{n} \leq a_{n}$. So $a_{n}=$ $b_{n}$.

Again, from the equation $b_{n+2}+b_{n+1} x=0$ we conclude that $b_{n+1} x=0$. Therefore, multiplying the equation $b_{n+1}+$ $b_{n} x=a_{n} x$ by the ideal $b_{n+1}$ we have

$$
b_{n+1}=b_{n+1}+b_{n} b_{n+1} x=a_{n} b_{n+1} x=b_{n} b_{n+1} x=0 .
$$

So, the given system of the principal ideal equations simplifies and we have

$$
\left\{\begin{array}{l}
b_{1}+x=a_{1}+x \\
b_{2}+b_{1} x=a_{2}+a_{1} x \\
\ldots \\
b_{n-1}+b_{n-2} x=a_{n-1}+a_{n-2} x \\
b_{n}+b_{n-1} x=a_{n}+a_{n-1} x \\
b_{n} x=a_{n} x
\end{array}\right.
$$

Again, we multiply the equation $b_{n-1}+b_{n-2} x=a_{n-1}+$ $a_{n-2} x$ by $a_{n-1}$ and hence obtain

$$
a_{n-1} b_{n-1}+a_{n-1} b_{n-2} x=a_{n-1}
$$

If we multiply by $x$ the equation $b_{n}+b_{n-1} x=a_{n}+a_{n-1} x$ we will obtain and hence $b_{n-1} x=a_{n-1} x$. Thus the equation

$$
a_{n-1}=a_{n-1} b_{n-1}+a_{n-1} b_{n-2} x
$$

implies that $a_{n-1} \leq b_{n-1}$. Similarly $b_{n-1} \leq a_{n-1}$. Therefore $a_{n-1}=b_{n-1}$. After the finite number of steps using the prescribed procedure we will have the following finally reduced system:

$$
\left\{\begin{array}{l}
b_{1}+x=a_{1}+x \\
b_{1} x=a_{1} x
\end{array}\right.
$$

But, multiplying the first equation by $a_{1}$ we obtain

$$
a_{1}=a_{1}+a_{1} x=a_{1} b_{1}+a_{1} x=a_{1} b_{1}+b_{1} x \leq b_{1} .
$$

Again, by the similar consideration we can conclude that $a_{1}=b_{1}$.

So, having SNFs of $A$ and $B$ such that $A \oplus x R \cong B \oplus x R$ we have obtained that the summand $x R$ can be cancellated and $A=B$ as was desired. The lemma is proved.

As a corollary we obtain the following result.

Theorem 1. Let $R$ be a morphic ring and $[A],[B] \in K_{0}^{\prime}(R)$. Then $[A]=[B]$ if and only if $A=B$ in case when $A, B$ are reduced to the $\mathrm{SNF}$, and $A \cong B$ in the other case.

Proof. Suppose that $[A]=[B]$. Then $A \oplus X \cong B \oplus X$ for some $X=x_{1} R \oplus \ldots \oplus x_{n} R$ by Lemma 1 . Let $A^{\prime}$ and $B^{\prime}$ be the SNFs of

$$
A \oplus\left(x_{1} R \oplus \ldots \oplus x_{n-1} R\right), B \oplus\left(x_{1} R \oplus \ldots \oplus x_{n-1} R\right)
$$

respectively. Then by the previous lemma $A^{\prime} \oplus x_{n} R \cong B^{\prime} \oplus$ $x_{n} R$ implies that $A^{\prime}=B^{\prime}$ and hence

$$
A \oplus\left(x_{1} R \oplus \ldots \oplus x_{n-1} R\right) \cong B \oplus\left(x_{1} R \oplus \ldots \oplus x_{n-1} R\right) .
$$

Continuing this process we will finally obtain that $A \cong B$. If $A$ and $B$ are are reduced to the SNF then $A=B$. The theorem is proved.

Now we need to formulate one well-known property of the tensor product of modules over the commutative ring.

Proposition 1. Let $M$ be a $R$-module and $I, J$ are some ideals of a commutative ring $R$. Then

(i) $M \otimes_{R} R / I \cong M / I M$;

(ii) $R / I \otimes_{R} R / J \cong R /(I+J)$.

In the following lemma we apply this Proposition in order to obtain one surprising property of the principal ideals of a morphic ring.

Lemma 3. For every pair of elements $a, b \in R$ of a morphic ring $R: a R \otimes_{R} b R \cong a R \cap b R$.

Proof. By the definition of a morphic ring and the mentioned above result we have that $a R \otimes_{R} b R \cong R / \operatorname{Ann}(a) \otimes_{R}$ $R / \operatorname{Ann}(b) \cong R /(\operatorname{Ann}(a)+\operatorname{Ann}(b)) \cong \operatorname{Ann}(\operatorname{Ann}(a)+$ $\operatorname{Ann}(b)) \cong a R \cap b R$ as was desired.

In the classical K-theoretical investigations the Grothendieck's group $K_{0}(R)$ can be considered as a ring if we assume that $R$ is a commutative ring and the product is defined as

$$
b_{n} x+b_{n-1} x=a_{n} x+a_{n-1} x
$$

$$
[P] \cdot[Q]=\left[P \otimes_{R} Q\right]
$$


for any finitely generated projective $R$-modules $P$ and $Q$ over a commutative ring $R$. In the similar manner we obtain

Theorem 2. Let $R$ be a commutative morphic ring. Then an additive abelian group $K_{0}^{\prime}(R)$ becomes a commutative ring with 1 if we define a product

$$
[a R][b R]=\left[a R \otimes_{R} b R\right],
$$

for any $a, b \in R$, and extend it on the arbitrary elements of $K_{0}^{\prime}(R)$ by the linearity.

Remark that any element any element of $K_{0}^{\prime}(R)$ can be written as $[A]-[B]$, where $A, B \in \Delta(R)$ are reduced to the $\mathrm{SNF}$ and there is no pair of terms $a_{i} R, b_{j} R$ such that it can be cancellated in the expression $[A]-[B]$.

Now we try to understand how behaves $K_{0}^{\prime}(R)$ under the base ring $R$ change and how one can describe its structure in the simplest case.

Proposition 2. $K_{0}^{\prime}$ is a functor from the category MorphicRings of the morphic rings to the Rings category.

Proof. Let $f: R \rightarrow R^{\prime}$ be a homomorphism of the morphic rings. For any element

$$
M=m_{1} R \oplus \ldots \oplus m_{n} R \in \Delta(R)
$$

one can define an element $M^{\prime}=f_{\sharp}(M)$ in the following manner:

$$
\begin{gathered}
f_{\sharp}(M)=R^{\prime} \otimes_{R} M \cong \bigoplus_{i=1}^{n}\left(R^{\prime} \otimes_{R} m_{i} R\right) \cong \\
\cong \bigoplus_{i=1}^{n}\left(R^{\prime} \otimes_{R} R / \mu_{i} R\right) \cong \bigoplus_{i=1}^{n}\left(R^{\prime} / f\left(\mu_{i}\right) R^{\prime}\right) \in \Delta\left(R^{\prime}\right) .
\end{gathered}
$$

Thus, a ring's map $f: R \rightarrow R^{\prime}$ rises a correspondence

$$
f_{*}: \begin{cases}K_{0}^{\prime}(R) & \rightarrow K_{0}^{\prime}\left(R^{\prime}\right) \\ {[A]-[B]} & \mapsto\left[f_{\sharp}(A)\right]-\left[f_{\sharp}(B)\right]\end{cases}
$$

of the abelian groups $K_{0}^{\prime}(R)$ and $K_{0}^{\prime}\left(R^{\prime}\right)$. Moreover, if $[a R],[b R] \in K_{0}^{\prime}(R)$ and additionally we set $g R=a R \cap$ $b R, g R \sim \gamma R, a R \sim \alpha R, b R \sim \beta R$ then

$$
\begin{gathered}
f_{*}\left(\left[a R \otimes_{R} b R\right]\right)=f_{*}([a R \cap b R])= \\
=\left[R^{\prime} \otimes_{R}(a R \cap b R)\right]=\left[R^{\prime} \otimes_{R}(g R)\right]= \\
=\left[R^{\prime} \otimes_{R} R / \gamma R\right]=\left[R^{\prime} / f(\gamma) R^{\prime}\right]=[\operatorname{Ann}(f(\gamma))]
\end{gathered}
$$

and

$$
\begin{gathered}
f_{*}([\operatorname{Ann}(a)]) \cdot f_{*}([\operatorname{Ann}(b)])=\left[R^{\prime} / f(\alpha) R^{\prime}\right] \cdot\left[R^{\prime} / f(\beta) R^{\prime}\right]= \\
=\left[R^{\prime} / f(\alpha) R^{\prime} \otimes_{R} R^{\prime} / f(\beta) R^{\prime}\right]=\left[R^{\prime} /\left(f(\alpha) R^{\prime}+f(\beta) R^{\prime}\right)\right]= \\
=\left[\operatorname{Ann}\left(f(\alpha) R^{\prime}+f(\beta) R^{\prime}\right)\right]=\left[\operatorname{Ann}\left(f\left(\alpha R^{\prime}+\beta R^{\prime}\right)\right)\right]= \\
=[\operatorname{Ann}(f(\gamma))] .
\end{gathered}
$$

So $f_{*}$ becomes a ring's homomorphism. Therefore

$$
K_{0}^{\prime} \text { : MorphicRings } \rightsquigarrow \text { Rings }
$$

is a map from the category MorphicRings of all morphic rings and their homomorphisms to the category Rings defined by the rule

$$
K_{0}^{\prime}:\left\{\begin{array}{ll}
R & \mapsto K_{0}^{\prime}(R) \\
R \stackrel{f}{\rightarrow} R^{\prime} & \mapsto K_{0}^{\prime}(R) \stackrel{f_{*}}{\longrightarrow} K_{0}^{\prime}\left(R^{\prime}\right)
\end{array} .\right.
$$

Then we need to verify: is it a functor or not? Indeed, if $f=1_{R}: R \rightarrow R$ then for any $[A]-[B] \in K_{0}^{\prime}(R)$ we obtain

$$
\begin{aligned}
& f_{*}([A]-[B])=\left[f_{\sharp}(A)\right]-\left[f_{\sharp}(B)\right]= \\
& =\left[R \otimes_{R} A\right]-\left[R \otimes_{R} B\right]=[A]-[B]
\end{aligned}
$$

and hence $K_{0}^{\prime}\left(1_{R}\right)=1_{K_{0}^{\prime}(R)}$. If $R \stackrel{f}{\rightarrow} R^{\prime} \stackrel{g}{\rightarrow} R^{\prime \prime}$ are two homomorphisms of the morphic rings then we need to prove that

$$
(g \circ f)_{*}=g_{*} \circ f_{*} .
$$

Without loss of the generality take any $[a R] \in K_{0}^{\prime}(R)$ and assume that $a R \sim \alpha R$. Then

$$
(g \circ f)_{*}([a R])=\left[R^{\prime \prime} \otimes_{R} a R\right]=\left[R^{\prime \prime} / g(f(\alpha)) R^{\prime \prime}\right]
$$

and

$$
g_{*}\left(f_{*}(a R)\right)=g_{*}\left(\left[R^{\prime} \otimes_{R} a R\right]\right)=g_{*}\left(R^{\prime} / f(\alpha) R^{\prime}\right)=
$$

$=\left[R^{\prime \prime} \otimes_{R^{\prime}} R^{\prime} / f(\alpha) R^{\prime}\right]=\left[R^{\prime \prime} / g(f(\alpha)) R^{\prime \prime}\right]=[\operatorname{Ann}(g(f(\alpha)))]$

as was desired. So, $K_{0}^{\prime}$ is a functor. The proposition is proved.

As a consequence it can be shown that $K_{0}^{\prime}$ preserves direct products of the morphic rings:

$$
K_{0}^{\prime}\left(\prod_{i} R_{i}\right) \cong \prod_{i} K_{0}^{\prime}\left(R_{i}\right)
$$

Theorem 3. Let $R$ be a morphic ring. Then $K_{0}^{\prime}(R)$ has a direct summand isomorphic to the ring of integers $\mathbb{Z}$.

Proof. Considering any maximal ideal $M$ of $R$ we define a natural homomorphism

$$
f: R \rightarrow F=R / M
$$

of a ring $R$ onto a field $F$. Then $K_{0}^{\prime}$ induces a homomorphism

$$
f_{*}: K_{0}^{\prime}(R) \rightarrow \mathbb{Z} \cong K_{0}^{\prime}(F)
$$

A map

$$
i_{*}: \begin{cases}\mathbb{Z} & \rightarrow K_{0}^{\prime}(R) \\ n & \mapsto n[R]\end{cases}
$$

is a monomorphism such that $f_{*} i_{*}=1_{\mathbb{Z}}$ and the following short exact sequence

$$
0 \rightarrow \operatorname{ker} f_{*} \rightarrow K_{0}^{\prime}(R) \stackrel{f_{*}}{\longrightarrow} \mathbb{Z} \rightarrow 0
$$

splits

$$
K_{0}^{\prime}(R) \cong \mathbb{Z} \oplus \operatorname{ker} f_{*} .
$$

The latter isomorphism proves the theorem.

Theorem 4. If $R$ is a morphic ring then $K_{0}^{\prime}(R) \cong \mathbb{Z}$ if and only if $R$ is a field.

Proof. By the previous theorem we know that $K_{0}^{\prime}(R) \cong \mathbb{Z}$ if and only if for any maximal ideal $M$ of a ring $R$

$$
\operatorname{ker} f_{*}=0
$$

where $f: R \rightarrow F=R / M$ is a natural homomorphism.

Suppose that ker $f_{*}=\left\{[A]-[B] \mid f_{*}([A])=f_{*}([B])\right\}=$ 0 . This means that whether $f_{*}([A])=f_{*}([B])$ then $[A]=$ $[B]$. Since $R$ is a morphic ring an element $A \in \Delta(R)$ can be considered in the form

$$
A=R / a_{1} R \oplus \ldots \oplus R / a_{n} R
$$


where $a_{1} R \subseteq \ldots \subseteq a_{n} R$. Then

$$
\begin{gathered}
f_{\sharp}\left(R / a_{i} R\right)=R / M \otimes_{R} R / a_{i} R \cong \\
\cong R /\left(M+a_{i} R\right)=\left\{\begin{array}{ll}
F, & a_{i} \in M \\
0, & a_{i} \notin M
\end{array} .\right.
\end{gathered}
$$

Hence $f_{*}([A])=k$, where $k \in\{1, \ldots, n\}$ is such that $a_{1}, a_{2}, \ldots, a_{k} \in M, a_{k+1}, \ldots, a_{n} \notin M$. Thus, the equality $f_{*}([A])=f_{*}([B])$ means that the chains of the ideals $a_{1} R \subseteq$ $\ldots \subseteq a_{n} R$ and $b_{1} R \subseteq \ldots \subseteq b_{m} R$ have the same number of ideals inside $M$. Therefore, the condition ker $f_{*}=0$ means that there is at most one chain of the ideals $a_{1} R \subseteq \ldots \subseteq a_{k} R$ of the length $k$ inside $M$ for any $k \geq 0$. But if we take a unique chain of the ideals of the length $k \geq 2$ inside $M$ then any term of this chain is itself a chain of the length 1 inside $M$. But all chains of the length 1 are equal, so there is at most one principal ideal $a R$ inside $M$. If $M \neq a R$ then there is $b \in M \backslash a R$ such that $a R \neq b R$. But this is impossible since $a R$ is a unique chain of the length 1 , so $M=a R$.

Moreover, if we take $[A]=[R \oplus R \oplus a R]$ and $[B]=[R \oplus a R]$ then $f_{*}([A])=f_{*}([B])=1$ and hence $[A]=[B]$. Then

$$
[R \oplus R \oplus a R]=[R \oplus a R]
$$

implies that $[R]=[0]$. By the Theorem 1 we obtain that $R=0$ that is a contradiction, and such maximal ideal $M$ cannot exist. After repeating the similar procedure to the other maximal ideals we obtain that there are no maximal ideals in $R$ and $R$ have to be a field. The theorem is proved.

\section{The representation of $K_{0}^{\prime}$ by the Witt vectors}

In the current section we will try to find a convenient way for the addition and multiplication of the elements of $K_{0}^{\prime}$.

Definition 5. A Witt ring (or Witt vectors) for a commutative ring $R$ is called a set

$$
W(R)=1+t R[[t]]=\left\{1+a_{1} t+a_{2} t^{2}+\ldots \mid a_{1}, a_{2}, \ldots \in R\right\}
$$

that is an abelian group under the multiplication operation between the formal power series (this operation represents the additive operation of a ring $W(R)$ ) and the ring multiplication operation is defined by the convolution rule in the following way: any $f(t) \in W(R)$ can be written as

$$
f(t)=\prod_{i=1}^{\infty}\left(1+r_{i} t\right)
$$

so for the arbitrary $r \in R$ we define

$$
(1+r t) * f(t)=f(r t)
$$

and extend this rule to the infinite products. An identity of a ring $W(R)$ is an element $1+t$ and 1 is a zero element.

Before applying the mentioned ring construction we need to formulate the following definition.

Definition 6. Let $R$ be a commutative Bezout ring. A semiring

$$
\Omega(R)=\{a R \mid a \in R\}
$$

of all its principal ideals under the addition and intersection of the ideals is called a globalization of a ring $R$.
If one take $R=\mathbb{Z}_{12}$ then $\Omega(R)=\{0,2 R, 3 R, 4 R, 6 R, R\}$. Other examples of globalizations can be obtained similarly. Thus, for any commutative Bezout ring $R$ we can consider the ring completion $G(R)=\Omega^{-1} \Omega(R)$ of its globalization $\Omega(R)$. Since $G(R)$ becomes a commutative ring with identity then we can define a subsemiring

$$
\begin{aligned}
W_{0}(G(R))= & \left\{1+\left(a_{1} R\right) t+\left(a_{2} R\right) t^{2}+\ldots+\left(a_{n} R\right) t^{n} \mid\right. \\
& \left.\mid a_{1} R \supseteq \ldots \supseteq a_{n} R, n \geq 0\right\}
\end{aligned}
$$

of a ring $W(G(R))$ considered as a semiring.

Any element $f(t)=1+\left(a_{1} R\right) t+\left(a_{2} R\right) t^{2}+\ldots+\left(a_{n} R\right) t^{n} \in$ $W_{0}(G(R))$ can be expressed in the form

$$
f(t)=\prod_{i=1}^{n}\left(1+a_{i} R t\right) .
$$

An identity element of $W_{0}(G(R))$ is $1+R t$ and 1 is its zero element. Furthermore, if

$$
f(t)=1+\sum_{i=1}^{n}\left(a_{i} R\right) t^{i}, g(t)=1+\sum_{j=1}^{m}\left(b_{j} R\right) t^{j}
$$

are any elements of $W_{0}(G(R))$ then their sum and product can be computed by the formulae

$$
\begin{gathered}
f(t) \cdot g(t)=1+\sum_{k=1}^{n+m}\left(\sum_{i+j=k}\left(a_{i} R \cap b_{j} R\right)\right) t^{k}, \\
f(t) * g(t)=\prod_{j=1}^{m} f\left(\left(b_{j} R\right) t\right)=\prod_{i=1}^{n} g\left(\left(a_{i} R\right) t\right)= \\
=\prod_{i, j=1,1}^{n, m}\left(1+\left(a_{i} R \cap b_{j} R\right) t\right) .
\end{gathered}
$$

After the direct computations one can conclude that the above definitions of the sum and product also belongs to $W_{0}(G(R))$.

Theorem 5. If $R$ is a morphic ring and $G(R)=\Omega^{-1} \Omega(R)$ then

$$
K_{0}^{\prime}(R) \cong W^{\prime}(G(R)),
$$

where $W^{\prime}(R)$ is a ring completion of a semiring $W_{0}(G(R))$.

Proof. In the following consideration the subtraction operation in the ring completion $W^{\prime}(G(R))$ will be denoted by $\frac{f(t)}{g(t)}$, for $f(t), g(t)$. In fact, it is a formal polynomial's division. So, the ring $W^{\prime}(R)$ can be described as

$$
\begin{gathered}
W^{\prime}(R)=\left\{\frac{1+\left(a_{1} R\right) t+\ldots+\left(a_{n} R\right) t^{n}}{1+\left(b_{1} R\right) t+\ldots+\left(b_{m} R\right) t^{m}} \mid\right. \\
\left.a_{1} R \supseteq \ldots \supseteq a_{n} R, b_{1} R \supseteq \ldots \supseteq b_{m} R, n, m \geq 0\right\}
\end{gathered}
$$

with the addition

$$
\frac{a(t)}{b(t)} \cdot \frac{c(t)}{d(t)}=\frac{a(t) c(t)}{b(t) d(t)}
$$

and multiplication

$$
\frac{a(t)}{b(t)} * \frac{c(t)}{d(t)}=\frac{(a(t) * c(t))(b(t) * d(t))}{(a(t) * d(t))(b(t) * c(t))}
$$

for any $a(t), b(t), c(t), d(t) \in W_{0}(G(R))$. We define a map

$$
F_{R}: \begin{cases}K_{0}^{\prime}(R) & \rightarrow W^{\prime}(G(R)) \\ {[A]-[B]} & \mapsto \frac{a(t)}{b(t)}=\frac{1+\sum_{i=1}^{n}\left(a_{i} R\right) t^{i}}{1+\sum_{j=1}^{m}\left(b_{j} R\right) t^{j}}\end{cases}
$$


where $A=a_{1} R \oplus \ldots \oplus a_{n} R, B=b_{1} R \oplus \ldots \oplus b_{m} R$ are reduced to the SNF. The map $F_{R}$ is a bijection since the SNF is defined uniquely and $a(t)$ and $b(t)$ have a uniquely determined decompositions

$$
a(t)=\prod_{i=1}^{n}\left(1+\left(a_{i} R\right) t\right), b(t)=\prod_{j=1}^{m}\left(1+\left(b_{j} R\right) t\right) .
$$

Also, it is a homomorphism since

$$
\begin{gathered}
F_{R}(([A]-[B])+([C]-[D]))=F_{R}([A \oplus C]-[B \oplus D])= \\
=\frac{a(t) c(t)}{b(t) d(t)}=F_{R}([A]-[B]) \cdot F_{R}([C]-[D])
\end{gathered}
$$

and

$$
\begin{gathered}
F_{R}(([A]-[B])([C]-[D]))= \\
=F_{R}\left(\left[A \otimes_{R} C\right]+\left[B \otimes_{R} D\right]-\left[B \otimes_{R} C\right]-\left[A \otimes_{R} D\right]\right)= \\
=\frac{\prod_{i, j}\left(1+\left(a_{i} R \cap c_{j} R\right) t\right) \prod_{k, l}\left(1+\left(b_{k} R \cap d_{l} R\right) t\right)}{\prod_{i, l}\left(1+\left(a_{i} R \cap d_{l} R\right) t\right) \prod_{k, j}\left(1+\left(b_{k} R \cap c_{j} R\right) t\right)}= \\
=\frac{(a(t) * c(t))(b(t) * d(t))}{(a(t) * d(t))(b(t) * c(t))}=\frac{a(t)}{b(t)} * \frac{c(t)}{d(t)}= \\
=F_{R}([A]-[B]) * F_{R}([C]-[D]) .
\end{gathered}
$$

Thus, $F_{R}$ is an isomorphism. The theorem is proved.

Proposition 3. The maps

$$
\begin{aligned}
& W^{\prime}: \text { Rings } \rightsquigarrow \text { Rings } \\
& \Omega: \text { BezoutRings } \rightsquigarrow \text { Semirings } \\
& G=\Omega^{-1} \Omega(R): \text { Rings } \rightsquigarrow \text { Rings }
\end{aligned}
$$

defined above are the functors.

Proof. If we set

$$
\begin{gathered}
W^{\prime}(f): \begin{cases}W^{\prime}(R) & \rightarrow W^{\prime}\left(R^{\prime}\right) \\
\frac{1+a_{1} t+\ldots+a_{n} t^{n}}{1+b_{1} t+\ldots+b_{m} t^{m}} & \mapsto \frac{1+f\left(a_{1}\right) t+\ldots+f\left(a_{n}\right) t^{n}}{1+f\left(b_{1}\right) t+\ldots+f\left(b_{m}\right) t^{m}}\end{cases} \\
\Omega(f): \begin{cases}\Omega(R) & \rightarrow \Omega\left(R^{\prime}\right) \\
a R & \mapsto f(a) R^{\prime}\end{cases} \\
G(f): \begin{cases}\Omega^{-1} \Omega(R) & \rightarrow \Omega^{-1} \Omega\left(R^{\prime}\right) \\
a R-b R & \mapsto f(a) R^{\prime}-f(b) R^{\prime}\end{cases}
\end{gathered}
$$

for any homomorphism $f: R \rightarrow R^{\prime}$ in the appropriate source category then images of $f$ such as $W^{\prime}(f), \Omega(f), G(f)$ are precisely the homomorphisms in the target categories of the given maps. The fact that $W^{\prime}, \Omega$ and $G$ preserves identity homomorphisms and the compositions can be shown by the routine calculations. So, $W^{\prime}, \Omega$ and $G$ are the functors.

Theorem 6. If we consider $\Omega$ as a functor $\Omega$ : MorphicRings $\rightsquigarrow$ Semirings then there is a natural equivalence of functors

$$
K_{0}^{\prime} \approx W^{\prime} G
$$

Proof. By Theorem $5 K_{0}^{\prime}(R) \cong W^{\prime}(G(R))$ for any morphic ring $R$ via the isomorphism $F_{R}$. So, if $f: R \rightarrow R^{\prime}$ is any homomorphism of morphic rings $R$ and $R^{\prime}$ then

$$
\begin{array}{ll}
K_{0}^{\prime}(R) \stackrel{F_{R}}{\longrightarrow} & W^{\prime}(G(R)) \\
\downarrow K_{0}^{\prime}(f) & \downarrow W^{\prime}(G(f)) \\
K_{0}^{\prime}\left(R^{\prime}\right) \stackrel{F_{R^{\prime}}}{\longrightarrow} & W^{\prime}\left(G\left(R^{\prime}\right)\right)
\end{array}
$$

is a commutative diagram since

$$
\begin{gathered}
\left(W^{\prime}(G(f)) \circ F_{R}\right)([A]-[B])= \\
=W^{\prime}(G(f))\left(\left(1+\sum_{i=1}^{n}\left(a_{i} R\right) t^{i}\right) /\left(1+\sum_{j=1}^{m}\left(b_{j} R\right) t^{j}\right)\right)= \\
=\left(1+\sum_{i=1}^{n}\left(f\left(a_{i}\right) R^{\prime}\right) t^{i}\right) /\left(1+\sum_{j=1}^{m}\left(f\left(b_{j}\right) R^{\prime}\right) t^{j}\right)= \\
=\left(F_{R^{\prime}} \circ K_{0}^{\prime}(f)\right)([A]-[B]) .
\end{gathered}
$$

Thus, $K_{0}^{\prime} \approx W^{\prime} G$ as was desired. The theorem is proved.

The latter result shows the way that one can compute the SNF of the block sum $A \oplus B$ and Kroneker's product $A \otimes B$ of two given matrices $A$ and $B$ that are already reduced to their SNF's. Since the multiplication in $K_{0}^{\prime}(R)$ can be done after some number of the addition operations (this follows from the distributivity of the tensor product over the direct sums) then naturally arises a question: are there any other way to represent the elements of $K_{0}^{\prime}(R)$ for more efficient evaluation of the sums of the given elements?

The answer is affirmative and below we give a solution. If $[A]=\left[a_{1} R \oplus \ldots \oplus a_{n} R\right]$ and $[B]=\left[b_{1} R \oplus \ldots \oplus b_{m} R\right]$ are the elements of $K_{0}^{\prime}(R)$ that are reduced to their SNF then we represent $[A]$ and $[B]$ in a form

$[X] \mapsto\left(\begin{array}{ccccccccc}x_{n} & x_{n-1} & \cdots & x_{1} & 1 & 0 & 0 & \cdots 0 & 0 \\ 0 & x_{n} & \cdots & x_{2} & x_{1} & 1 & 0 & \cdots 0 & 0 \\ \cdots & \cdots & \cdots & \cdots & \cdots & \cdots & \cdots & \cdots & \cdots \\ 0 & 0 & \cdots & 0 & 0 & 0 & 0 & \cdots x_{n} & x_{n-1} \\ 0 & 0 & \cdots & 0 & 0 & 0 & 0 & \cdots 0 & x_{n}\end{array}\right)$

$\in M_{n+m}(\Omega(R))$

and multiply the respective matrices for $[A]$ and $[B]$ in $M_{n+m}(\Omega(R))$, then the resulting matrix will represent the sum $[A \oplus B]$. In other words: if $J$ is a Jordan matrix in $M_{n+m}(\Omega(R))$ with the zero eigenvalue then

$$
\begin{aligned}
& {[A] \leftrightarrow a_{n} E+a_{n-1} J+\ldots+a_{1} J^{n-1}+J^{n}} \\
& {[B] \leftrightarrow b_{n} E+b_{m-1} J+\ldots+b_{1} J^{m-1}+J^{m}}
\end{aligned}
$$

$[A \oplus B] \leftrightarrow\left(a_{n} E+\ldots+a_{1} J^{n-1}+J^{n}\right)\left(b_{n} E+\ldots+b_{1} J^{m-1}+J^{m}\right)$ and the latter product will be the necessary result. On the other hand, it is not necessary to multiply pairwise every row and column in order to obtain the result. In fact, the situation can be solved even simpler - the product

$$
\left(\begin{array}{c}
c_{1} \\
c_{2} \\
\vdots \\
c_{n+m}
\end{array}\right)=T \times\left(\begin{array}{c}
0 \\
\vdots \\
0 \\
1 \\
b_{1} \\
\vdots \\
b_{m-1} \\
b_{m}
\end{array}\right)
$$

represents the sum $[C]=[A \oplus B]$ in the SNF, where $T$ is a matrix whose rows are shifts of a row $\left(a_{n}, a_{n-1}, \cdots, a_{1}, 1,0, \cdots 0\right)$. 


\section{The internal properties of $K_{0}^{\prime}$}

Lemma 4. Every idempotent $e$ of $K_{0}^{\prime}(R)$ can be written in the following form

$$
e=[a R]([R]-[b R]),
$$

for some $a, b \in R$.

Proof. Let $[A]-[B]$ be some idempotent of $K_{0}^{\prime}(R)$ that is written in the reduced form (i.e. $A, B$ are reduced to the SNF and there is no pair of $a_{i} R, b_{j} R$ that can be cancellated). Then $([A]-[B])^{2}=[A]-[B]$ and thus

$$
[A]^{2}+[B]^{2}+[B]=[A]+2[A][B] .
$$

Suppose that $A^{\prime}=a_{2} R \oplus \ldots \oplus a_{n} R \neq 0$. Hence $[A]^{2}=$ $\left[a_{1} R \oplus A^{\prime}\right][A]=[A]+\left[A^{\prime}\right][A]$ and so

$$
[A]^{2}+[B]^{2}+[B]=[A]+2[A][B] .
$$

Since the highest terms of the LHS and RHS have to be equal then $a_{1} R \cap a_{2} R+b_{1} R=a_{1} \cap b_{1}$ and hence $a_{2} R \subseteq b_{1} R, a_{2} R=$ $a_{1} R \cap b_{1} R$. Moreover, if $A^{\prime \prime}=a_{3} R \oplus \ldots \oplus a_{n} R$ then

$$
\begin{gathered}
{\left[A^{\prime}\right][A]=\left[a_{2} R \oplus A^{\prime \prime}\right]\left[a_{1} R \oplus a_{2} R \oplus A^{\prime \prime}\right]=} \\
=\left[a_{2} R \oplus a_{2} R \oplus 3 A^{\prime \prime}\right]+\left[A^{\prime \prime}\right]^{2}=\ldots= \\
=\left[2 a_{2} R \oplus 4 a_{3} R \oplus \ldots \oplus(2 n-2) a_{n} R\right], \\
{[B]^{2}+[B]=\left[2 b_{1} R \oplus 4 b_{2} R \oplus \ldots \oplus(2 m)\left(b_{m} R\right)\right] .}
\end{gathered}
$$

Therefore

$$
\begin{gathered}
\sum_{k=1}^{m} 2 k\left[b_{k} R\right]+\sum_{i=2}^{n}(2 i-2)\left[a_{i} R\right]=2\left[a_{1} R \cap b_{1} R\right]+ \\
+2 \sum_{i=2}^{n}\left[a_{i} R \cap b_{1} R\right]+2 \sum_{k=2}^{m}\left[a_{1} R \cap b_{k} R\right]+2 \sum_{i, k=2}^{n, m}\left[a_{i} R \cap b_{k} R\right] .
\end{gathered}
$$

But $a_{i} R \cap b_{1} R=a_{i} R$, for $2 \leq i \leq n$ and $a_{2} R=a_{1} R \cap b_{1} R$, so cancellating both parts we have

$$
\begin{gathered}
\sum_{k=1}^{m} 2 k\left[b_{k} R\right]+\sum_{i=3}^{n}(2 i-4)\left[a_{i} R\right]=2 \sum_{i=2}^{n}\left[a_{i} R \cap b_{2} R\right]+ \\
+2 \sum_{k=2}^{m}\left[a_{1} R \cap b_{k} R\right]+2 \sum_{i=2, k=3}^{n, m}\left[a_{i} R \cap b_{k} R\right] .
\end{gathered}
$$

Again, using the equality of the highest terms we have that $b_{1} R+a_{3} R=a_{1} R \cap b_{2} R$ and hence $b_{1} R \subseteq a_{1} R, b_{1} R=b_{2} R$. Using the fact that $a_{1} R \cap B_{k} R=b_{k} R$, for $1 \leq k \leq m$ we can obtain a simplification

$$
\begin{aligned}
4\left[b_{2} R\right] & +\sum_{k=3}^{m}(2 k-2)\left[b_{k} R\right]+\sum_{i=4}^{n}(2 i-6)\left[a_{i} R\right]= \\
& =2\left[a_{2} R\right]+2 \sum_{i=2, k=3}^{n, m}\left[a_{i} R \cap b_{k} R\right] .
\end{aligned}
$$

The equality of the highest terms implies that $a_{2} R=b_{1} R=$ $b_{2} R$ and hence the combination $[A]-[B]$ is not written in the reduced form. The obtained contradiction implies that $A^{\prime}=0$ and $A=a R$, for some $a \in R$. Then $([a R]-[B])^{2}=[a R]-[B]$ implies that

$$
[a R]+[B]^{2}+[B]=[a R]+2[a R][B]
$$

and hence

$$
\sum_{k=1}^{m}(2 k)\left[b_{k} R\right]=2 \sum_{k=1}^{m}\left[a R \cap b_{k} R\right]
$$

. Since the both parts of the latter equality are already written in the SNF then $b_{1} R=a R \cap b_{1} R$. Therefore $b_{1} R \subseteq a R$ and hence

$$
\sum_{k=1}^{m}(2 k)\left[b_{k} R\right]=2 \sum_{k=1}^{m}\left[b_{k} R\right] .
$$

The number of terms in the LHS is $m^{2}+m$ and in the RHS is $2 \mathrm{~m}$. Since we have assumed that $[B]$ is in the reduced form then the only possible case is $m^{2}+m=2 m$ and hence $m=1$.

So, any idempotent $[A]-[B]$ can be written as $[A]-[B]=$ $[a R]-[a R \cap b R]$, where $a R \supseteq b R$ or equivalently

$$
[A]-[B]=[a R]([R]-[b R]) .
$$

The lemma is proved.

Lemma 5. $[A]$ is an invertible element of $K_{0}^{\prime}(R)$ if and only if $[A]=[R]$.

Proof. Let $[A]([C]-[D])=[R]$ and $[C]-[D]$ is written in the reduced form. Then $[A][C]=[R]+[A][D]$ and hence the equality of the highest terms implies that $a_{1} R \cap c_{1} R=R$. The latter implies that $a_{1} R=c_{1} R=R$. Suppose that $A=$ $R \oplus A^{\prime}, C=R \oplus C^{\prime}, C^{\prime} \neq 0$. Then

$$
\left[A^{\prime}\right]+\left[C^{\prime}\right]+\left[A^{\prime}\right]\left[C^{\prime}\right]=[D]+\left[A^{\prime}\right][D]
$$

implies that $a_{2} R+c_{2} R=d_{1} R$. Let $A^{\prime}=a_{2} R \oplus A^{\prime \prime}, C^{\prime}=$ $c_{2} R \oplus C^{\prime \prime}, D=\left(a_{2} R+c_{2} R\right) \oplus D^{\prime}$.

After the substitution we will obtain

$$
\begin{gathered}
{\left[a_{2} R\right]+\left[A^{\prime \prime}\right]+\left[c_{2} R\right]+\left[C^{\prime \prime}\right]+\left[a_{2} R \cap c_{2} R\right]+\left[a_{2} R\right]\left[C^{\prime \prime}\right]+} \\
+\left[c_{2} R\right]\left[A^{\prime \prime}\right]+\left[A^{\prime \prime}\right]\left[C^{\prime \prime}\right]=\left[a_{2} R+c_{2} R\right]+\left[D^{\prime}\right]+ \\
+\left[a_{2} R+c_{2} R\right]\left[A^{\prime}\right]+\left[a_{2} R+c_{2} R\right]\left[D^{\prime}\right]
\end{gathered}
$$

and hence

$$
\begin{gathered}
{\left[a_{2} R+c_{2} R\right]+2\left[a_{2} R \cap c_{2} R\right]+\left[A^{\prime \prime}\right]+\left[C^{\prime \prime}\right]+\left[a_{2} R\right]\left[C^{\prime \prime}\right]+} \\
+\left[c_{2} R\right]\left[A^{\prime \prime}\right]+\left[A^{\prime \prime}\right]\left[C^{\prime \prime}\right]= \\
=\left[a_{2} R+c_{2} R\right]+2\left[D^{\prime}\right]+\left[a_{2} R\right]+\left[A^{\prime \prime}\right] .
\end{gathered}
$$

After the cancellation of the equal terms in the both sides we will have

$$
\begin{aligned}
2\left[a_{2} R \cap c_{2} R\right]+\left[C^{\prime \prime}\right]+ & {\left[a_{2} R\right]\left[C^{\prime \prime}\right]+\left[c_{2} R\right]\left[A^{\prime \prime}\right]+\left[A^{\prime \prime}\right]\left[C^{\prime \prime}\right]=} \\
& =2\left[D^{\prime}\right]+\left[a_{2} R\right] .
\end{aligned}
$$

Then $a_{2} R \cap c_{2} R=a_{2} R+d_{2} R$ and $d_{2} R \subseteq a_{2} R$. Moreover, $a_{2} R \cap c_{2} R=a_{2} R$ and $a_{2} R \subseteq c_{2} R$. Thus we conclude that $d_{1} R=a_{2} R+c_{2} R=c_{2} R$ and so the combination $[C]-[D]$ is not written in the reduced form. The obtained contradiction implies that $C^{\prime}=0$ and $c_{2} R=0$. Thus, $\left[A^{\prime}\right]=\left[A^{\prime}\right][D]+[D]$ and hence $a_{2} R=d_{1} R$. Therefore, $\left[a_{2} R\right]+\left[A^{\prime \prime}\right]=\left(\left[a_{2} R\right]+\right.$ $\left.\left[A^{\prime \prime}\right]\right)\left(\left[a_{2} R\right]+\left[D^{\prime}\right]\right)+\left[a_{2} R\right]+\left[D^{\prime}\right]$ implies that $\left[a_{2} R\right]+2\left[D^{\prime}\right]+$ $\left[A^{\prime \prime}\right]\left[D^{\prime}\right]=0$ and hence $a_{2} R=0$. As a result we have that $[A]=[R]$.

Lemma 6. If the highest terms of $[A],[B] \in K_{0}^{\prime}(R)$ are coprime and have zero intersection then $[A]-[B] \in U\left(K_{0}^{\prime}(R)\right)$ if and only if $[A]-[B]=[R]-2[x R]$, for some $[x R] \in K_{0}^{\prime}(R)$. 
Proof. Suppose that $[A]-[B] \in U\left(K_{0}^{\prime}(R)\right)$. Then $[A]^{2}+$ $[B]^{2}=([A]-[B])^{2} \in U\left(K_{0}^{\prime}(R)\right)$ and by Lemma $5[A]^{2}+$ $[B]^{2}=[R]$. The first and the second terms of $[A]^{2}+[B]^{2}$ are $a_{1} R+b_{1} R, a_{2} R+a_{1} R b_{1} R+b_{2} R$ and by Theorem 1 we obtain that $a_{1} R+b_{1} R=R$ and $a_{2} R+b_{2} R=a_{2} R+a_{1} R b_{1} R+b_{2} R=$ 0 . Then $a_{2} R=b_{2} R=0$ and $[A]-[B]=[a R]-[b R]$, for some coprime elements $a, b \in R$. So, $[A]-[B]=[a R \oplus b R]-2[b R]=$ $[(a R+b R) \oplus(a R \cap b R)]-2[b R]=[R]-2[b R]$. The lemma is proved.

Proposition 4. If $R$ is a morphic ring then $K_{0}^{\prime}(R)$ is a reduced ring, that is there are no nonzero nilpotent elements.

Proof. Suppose that $[A]-[B] \in K_{0}^{\prime}(R)$ is a nilpotent element such that

$$
([A]-[B])^{2}=[0] .
$$

Then $[A]^{2}+[B]^{2}=2\left[A \otimes_{R} B\right]$. By Theorem 1 this equality is equivalent to the fact that SNF of $\left(A \otimes_{R} A\right) \oplus\left(B \otimes_{R} B\right)$ and $\left(A \otimes_{R} B\right) \oplus\left(A \otimes_{R} B\right)$ are equal. But the highest term of the right hand side is $a_{1} R \cap b_{1} R$ and $a_{1} R+b_{1} R$ of the left hand side. Then

$$
\begin{aligned}
& a_{1} R, b_{1} R \subseteq a_{1} R+b_{1} R= \\
& =a_{1} R \cap b_{1} R \subseteq a_{1} R, b_{1} R .
\end{aligned}
$$

Hence $a_{1} R=b_{1} R$ and they can be cancellated in the expression $[A]-[B]$. Continuing this process we obtain that $[A]-[B]$ is simply $[X]=\left[x_{1} R \oplus \ldots \oplus x_{k} R\right]$ such that $[X]^{2}=0$.

But the highest term of $[X]^{2}$ is $x_{1} R$ and it is zero, so the whole $[X]$ and $[A]-[B]$ are $[0]$. So, $K_{0}^{\prime}(R)$ is a reduced ring. The proposition is proved.

In a case when $R$ is a commutative von Neumann regular ring the structure of a ring $K_{0}^{\prime}(R)$ becomes rather simple. Suppose that $a \in R$. Then there is $x \in R$ such that $a^{2} x=a$ and as can easily see

$$
a R \sim(1-a x) R
$$

Then

$$
\begin{gathered}
a R+\operatorname{Ann}(a)=R, a R \cap \operatorname{Ann}(a)=a R \cap(1-a x) R= \\
=a R \cdot(1-a x) R=0 .
\end{gathered}
$$

Thus, if $[A]-[B] \in K_{0}^{\prime}(R)$ then

$$
\begin{gathered}
{[A]-[B]=\left[\bigoplus_{i=1}^{n} a_{i} R\right]+\left[\bigoplus_{j=1}^{m} \operatorname{Ann}\left(b_{j}\right)\right]-} \\
-\sum_{j=1}^{m}\left[b_{j} R \oplus \operatorname{Ann}\left(b_{j}\right)\right]=\left[A^{\prime}\right]-m[R]
\end{gathered}
$$

for some $\left[A^{\prime}\right] \in K_{0}^{\prime}(R)$. In other words, we have obtained the following result.

Proposition 5. If $R$ is a commutative von Neumann regular ring then

$$
K_{0}^{\prime}(R)=\left\{[A]-m[R] \mid m \geq 0, A=a_{1} R \oplus \ldots \oplus a_{n} R\right\} .
$$

The latter result is rather important since it has a connection with the usual Grothendieck's group $K_{0}(R)$ of a von Neumann regular ring $R$.

Theorem 7. If $R$ is a commutative von Neumann regular ring then

$$
K_{0}(R)=K_{0}^{\prime}(R)
$$

Proof. By $[2,7,5,8]$ we know that

1 ) every finitely generated projective $R$-module is a finitely presented;

2) every finitely presented flat $R$-module is a projective;

3 ) every $R$-module over a von Neumann regular ring is flat

4) every commutative von Neumann regular ring is an elementary divisor ring

5 ) a commutative Bezout ring $R$ is an elementary divisor ring if and only if any finitely generated $R$-module can be decomposed in a finite direct sum of some cyclic $R$-modules.

So, any $A=a_{1} R \oplus \ldots \oplus a_{n} R$ is a projective module since it is flat and any finitely generated projective module $P$ can be decomposed in a finite direct sum of some cyclic modules. According to the construction of $K_{0}(R)$ and $K_{0}^{\prime}(R)$ they have to coincide. The theorem is proved.

It is known that for any projective module $P$ over a ring $R$ there are some free $R$-module $F$ and a submodule $Q$ of $F$ such that

$$
P \oplus Q=F \text {. }
$$

Thus as any element of $K_{0}^{\prime}(R)$ can be expressed in form $\left[P_{1}\right]-$ $\left[P_{2}\right]$ then

$$
\left[P_{1}\right]-\left[P_{2}\right]=\left[P_{1} \oplus Q_{2}\right]-\left[P_{2} \oplus Q_{2}\right]=\left[P_{1} \oplus Q_{2}\right]-n[R],
$$

where $P_{2} \oplus Q_{2} \cong R^{N}$ and we conclude that the studying of the structure of $K_{0}^{\prime}(R)$ help to understand the Grothendieck's group $K_{0}(R)$.

\section{REFERENCES}

[1] Bass H. K-theory and stable algebra, Publ.Math., 1964, 22, p.5-60.

[2] Bourbaki N. Algèbre, Ch. X, Masson, Paris, 1980.

[3] Golan J.S. Semirings and their applications, Kluwer Academic Publishers, Dordrecht, 1999, 381p.

Goodearl K.R. Von Neumann Regular Rings, Pitman, London, 1979, 369p.

[4] Goodearl K.R. Von Neumann Regular Rings, Pitman, London, 1979, 369p.

[5] Henriksen M. On a class of regular rings that are elementary divisor rings, Arch. Math. (Basel), 1973, 24, p.133-141.

[6] Kaplansky I. Elementary divisors and modules, Trans. Amer. Math. Soc., 1949, 66, p.464-491.

[7] Lambek J. Lectures on rings and modules, Blaisdell Publishing, 1966.

[8] Larsen M., Levis W., Shores T. Elementary divisor rings and finitely presented modules, Trans. Amer. Math. Soc., 1974, 187 , p.231-248.

[9] Milnor J.W. Introduction to algebraic K-theory, Annals of Mathematics Studies, 72, 1971.

[10] Nicholson W.K., Sanchez Campos E. Rings with the dual of the isomorphism theorem, J.Algebra, 2004, 271, p.391-406.

[11] Shores T. Modules over semihereditary Bezout rings, Proc. Amer. Math. Soc., 1974, 46.2, p.211-213.

[12] Vasserstein L.N. The stable rank of rings and dimensionality of topological spaces, Functional Anal. Appl., 1971, 5, p.102110 . 
[13] Zabavsky B.V. Diagonal reduction of matrices over rings, Mathematical Studies, Monograph Series. VNTL Publishers, 2012, volume XVI, 251p.

[14] Zabavsky B.V. Diagonal reduction of matrices over finite stable range rings, Mat.Stud., 2014, 41.1, p.101-108. 